Revue d'histoire de l'Amérique française

ZWB REVUE D.HISTOIRE DE L'AMÉRIQUE FRANÇAISE

\title{
Bibliographie de l'abbé Alexis Pelletier
}

\section{Thomas Charland}

Volume 1, numéro 3, décembre 1947

URI : https://id.erudit.org/iderudit/801405ar

DOI : https://doi.org/10.7202/801405ar

Aller au sommaire du numéro

Éditeur(s)

Institut d'histoire de l'Amérique française

ISSN

0035-2357 (imprimé)

1492-1383 (numérique)

Découvrir la revue

Citer ce document

Charland, T. (1947). Bibliographie de l'abbé Alexis Pelletier. Revue d'histoire de l'Amérique française, 1(3), 463-468. https://doi.org/10.7202/801405ar d'utilisation que vous pouvez consulter en ligne.

https://apropos.erudit.org/fr/usagers/politique-dutilisation/ 


\section{BIBLIOGRAPHIE 1 DE L'ABBÉ ALEXIS PELLETIER}

\section{A - BROCHURES}

1. Mgr. Gaume, sa thèse et ses defenseurs. Les classiques chrétiens et les classiques payens dans l'enseignement. St. Hyacinthe: de l'atelier typographique de Lussier et frère, propriétaires du "Courrier de St-Hyacinthe". 1865, 33p., $21 \mathrm{~cm}$.

2. Situation du monde actuel. Coup d'œil sur l'origine et la propagation du mal dans la societé, ou développement des principales idées contenues dans le discours de Mgr. Filippi, évêque d'Aquila, prononcé à l'Académie de la Religion Catholique à Rome, le 1er septembre 1864. [s.1.] 1865, 96p., $21 \mathrm{~cm}$.

3. La Question des classiques en présence des rectifications et des critiques de M. l'abbé Chandonnet, par un "chrétien ". [s.1.] 1865, 44p., $22.5 \mathrm{~cm}$.

4. La méthode chrêtienne considérée dans ses avantages et sa nécessité et réponses à certaines difficultés, par George Saint-Aimé. Ottawa, imprimé par G.-E. Desbarats, 1866, 51 p., $22 \mathrm{~cm}$.

5. Lettre à Monseigneur Baillargeon, évêque de Tloa, sur la question des classiques et commentaire sur la lettre du cardinal Patrizi, par George St.-Aimé. [s.1.n.d.] [1867] 51p., $22.5 \mathrm{~cm}$.

N.B. - Réimpression, 39p., $20.5 \mathrm{~cm}$.

6. Réponse aux dernières attaques dirigées par $M$. l'abbé Chandonnet contre les partisans de la méthode chrétienne et commentaires sur des documents authentiques qui dévoilent les machinations de MM. les abbés Chandonnet et Benjamin Paquet, par George Saint-Aimé. [s.1.] 1868, 56p., 21.5cm.

7. Quelques observations critiques sur l'ouvrage de M. l'abbé B. Paquet intitule: Le Libéralisme, par $A$. de $F$. Montréal: des presses à vapeur du journal Le Nouveau Monde, No 30, rue St. Gabriel, $1872,21 \mathrm{p} ., 24 \mathrm{~cm}$.

N.B. - Couverture: Le Libéralisme catholique ou observations critiques sur l'opuscule de M. l'abbé Benjamin Paquet intitule: Le Libéralisme, par $A$. de $F$.

Reproduction d'articles parus sous le titre d'Observations critiques, dans le Nouveau-Monde: $3,5,6,8,12,13,15,17,19$ et 22 juillet 1872 .

1. Cette "Bibliographie " se rattache à l'article: Un gaumiste canadien: l'abbé Alexis Pelletier, par Thomas Charland, (Revue d'histoire de l'Amérique frangaise, vol. 1 , no 2 , p. 195-236). 
8. La Question de l'Université, par Luigi. Montréal, Imprimerie " Le Franc-Parleur ", 22 , rue St. Gabriel, 1872 p. $51-60,21 \mathrm{~cm}$.

N.B. - En appendice à la Réponse au factum intitulé "Quelques remarques sur l'Université-Laval (novembre 1872) "), par la Rédaction $d u$ Franc-Parleur. Reproduction de deux articles du Franc-Parleur, 30 nov. et 7 déc. 1872 .

9. Les Quatre lettres. Croquis de topographie universitaire, par l'abbé Ste. Foi. Montréal, Imprimerie « Le Franc-Parleur », 22, rue St. Gabriel, 1872, 20 p., 21cm.

N.B. - Reproduction de trois articles du Franc-Parleur, 14, 21 et 28 déc. 1872.

10. Une conversation faisant suite à la brochure "Les Quatre lettres ", par l'abbé Ste. Foi. Montréal, Imprimerie "Le Franc-Parleur ", 22, rue St. Gabriel, 24 décembre 1872, p. 21-31, $20 \mathrm{~cm}$.

N.B. - Reproduit dans deux articles du Franc-Parleur, 4 et 11 janv. 1873.

11. Il y a du libéralisme et du gallicanisme en Canada, par Luigi (de la Rédaction du Franc-Parleur). Montréal, Imprimerie "Le Franc-Parleur », 22, rue St. Gabriel 1873, 45p., $21.5 \mathrm{~cm}$.

N.B. - Reproduction d'articles du Franc-Parleur, 25 janvier; 1, 8, 22 fév. et 1er mars 1873 .

12. Du Modérantisme ou de la fausse modération, par Luigi (de la Rédaction du FrancParleur). Montréal, Imprimerie "Le Franc-Parleur ", 22, rue St. Gabriel, 1873. $82 \mathrm{p} .21 \mathrm{~cm}$.

N.B - Reproduction d'articles du Franc-Parleur, 29 mars; 5, 19, 26 avril; 3, 6, 16, 27 mai; 3, 10, 17 et 20 juin 1873 .

13. Le Don Quichotte montrealais sur sa rossinante ou M. Dessaulles et la Grande Guerre Ecclésiastique, par Luigi. Montréal, Publié par la Société des Écrivains catholiques, $1873,101 \mathrm{p} ., 20.5 \mathrm{~cm}$.

N.B. - Le début fut reproduit dans un article du Franc-Parleur, 22 juillet 1873.

14. La Reforme chrétienne des études classiques, par un collaborateur du "FrancParleur " [par Luigi]. Montréal, Typ. Le Franc-Parleur N. 22, rue SaintGabriel, 1875, 194p., $20.5 \mathrm{~cm}$.

N.B. - Reproduction d'articles du Franc-Parleur, 17, 20, 24, 27 nov.; $11,15,18,23,31$ déc. $1874 ; 8,13,15,19,23,28,30$ janv.; 2,5 , $10,13,17,20,25,27$ fév.; 3 et 6 mars 1875 . 
15. M. l'abbé Sax et ses souffleurs ou Refutation des Erreurs contenues dans le Manifeste Libéral Québecquois, par un conservateur catholique. [s.1.] [Montréal, Imprimerie " Le Franc-Parleur )] 1875, 42p., $20 \mathrm{~cm}$.

N.B. - Reproduction d'articles du Franc-Parleur, 31 août, 3, 8, 10 et 14 sept. 1875 .

16. Coup d'œil sur le libéralisme européen et sur le libéralisme canadien. Démonstration de leur Parfaite Identité, [par Luigi] Montréal, Typ. Le Franc-Parleur, 22 , rue St. Gabriel 1876,79 p. $20 \mathrm{~cm}$.

N.B. - Reproduction d'articles du Franc-Parleur, 18, 21, 25, 26 avril, 2, 5, 9, 12, 16, 24, 30 mai; 9,14 et 21 juin 1876 .

17. La Source du Mal de l'Epoque au Canada, par un catholique. [s.1.n.d.] [1881],IV116p. $22 \mathrm{~cm}$.

\section{B - ARTICLES DE JOURNAUX}

\section{La GaZette des campagnes (Sainte-Anne de La Pocatik̀re)}

N.B. - De 1868 à 1871, l'abbé Pelletier à rédigé régulièrement la Revue de la Semaine, à laquelle il a parfois ajouté des articles, en gardant toujours l'anonymat.

\section{Le Nouveau-Monde (Montreal)}

Observations critiques - A. de F. 3,5,6,8,12,13,15,17,19 et 22 juillet 1872.

La Question des classiques clairement résolue - A. de F. 31 aout 1872.

Aux timorés du "Canadien" - Satis. 12 nov. 1872.

L'Article de la "Civilta catholica " - Alexis Pelletier, Ptre. 14 nov. 1872.

Le " Canadien » à propos de la "Civilta "-Alexis Pelletier, Ptre. 19 nov. 1872.

Le "Canadien" - Alexis Pelletier, Ptre. 21 nov. 1872.

La Lettre de Mgr l'archevêque - Justitia. 28 nov. 1872.

Correspondance-Justitia. 29 nov. 1872.

Une page d'histoire. St Sulpice et le Séminaire de Québec - Justitia. 3 déc. 1872.

Explication - Justitia. 11 déc. 1872.

La " Minerve » - Ju'stitia. 13 déc. 1872.

\section{Le Franc-Parleur (Montréal)}

Les Laches de notre epoque - Luigi. 9 nov. 1872.

A M. le Rédacteur du "Canadien " - Alexis Pelletier, Ptre. 16 nov. 1872.

Le Saint-Siège - L'abbé Ste.Foi. 23 et 30 nov. 1872.

La "Minerve " - Luigi. 23 nov. 1872. 
La Question de l'Université - Luigi. 30 nov. et 7 déc. 1872.

N.B. - Reproduits en appendice à la brochure Réponse au factum intitule "Quelques remarques sur l'Université-Laval », par la RÉdaction $d u$ Franc-Parleur, p. 51-60.

Les Quatre lettres - L'Abbé Ste. Foi. 14, 21 et 28 déc. 1872.

N.B. - Mis en brochure: Les Quatre lettres. Croquis de topographie universitaire.

Humilité et obeissance - Luigi. 24 déc. 1872.

Réforme chrétienne de l'enseignement - Luigi. 21 déc. 1872.

Une conversation faisant suite à la brochure Les quatre lettres - L'abbé Ste. Foi. 4 et 11 janv. 1873.

N.B. - Mis en brochure.

(A propos d'histoire) - (Suffit). 4 janv. 1873.

Il y a du libéralisme et du gallicanisme en Canada-Luigi. 25 janv., 1, 8, 22 fév. et ler mars 1873.

N.B. - Mis en brochure.

M. le G. V. Raymond et le libéralisme catholique - Justitia. 1er fév. 1873.

Mélanges - Luigi. 8 fév. 1873.

L'opuscule de M. l'abbe Paquet sur le liberalisme - Alexis Pelletier, Ptre. 15 fév. 1873.

Les écrivains catholiques - Luigi. 8 mars 1873.

Le Témoin $d u$ Journal de Québec - Luigi. 15 mars 1873.

Comment la "Minerve " apprécie M. Cauchon - Luigi. 22 mars 1873.

Du Modérantisme ou De la Fausse Modération - Luigi. 29 mars, 5, 19, 26 avril; $3,6,16,27$ mai; $3,10,17$ et 20 juin 1873.

N.B. - Mis en brochure.

La Minerve et Scavini - Luigi. 12 avril 1873.

Perversité de la "Minerve " - Luigi. 19 avril 1873.

Encore la note de Scavini - Luigi. 26 avril 1873.

Mgr Audisio cité par M. l'abbé Benjamin Paquet - Luigi. 21 mai 1873.

Le Sieur Hector Langevin - Luigi. 30 mai 1873.

Jugement du St. Office sur la dernière lecture de M. le G. V. Raymond-Luigi. 10 juin 1873.

Le Naturalisme ou la grande erreur moderne - Luigi. 8, 12, 22, 26, 29 juillet; 1er, $5,8,22$ et 29 aout 1873 .

A l'hon. L. A. Dessaulles. 22 Juillet 1873.

N.B. - début de la brochure Le Don Quichotte montréalais sur sa Rossinante.

Comme c'est honteux!!! - Luigi. 19 aodt 1873.

M. Dessaulles pose en victime - Luigi. 19 sept. 1873.

Encore d propos de M. Dessaulles - Luigi. ler et 3 oct. 1873.

L'examen critique d'un Faillible - Luigi. 10 oct. 1873.

Assemblee politique à Ste. Rose - Luigi. 17 oct. 1873.

La Question de l'amnistie - Luigi. 14 avril 1874.

Nos journaux - Luigi. 27 oct. 1874.

Deux élections - Luigi. 3 nov. 1874.

A propos de la condamnation de Lépine - Luigi. 6 nov. 1874.

Ce qui sortira du mal actuel - Luigi. 13 nov. 1874. 
Les Hommes nous manquent et pourquoi - Luigi. 17 nov. 1874.

Cet article et ceux qui suivirent: $20,24,27$ nov.; $11,15,18,23,31$ déc,; 8,13 , $15,19,23,28,30$ janv.; 2, 5, 10, 13, 17, 20, 25, 27 fév.; 3 et 6 mars 1875, furent mis en brochure sous le titre: La Reforme chretienne des études classiques, par un collaborateur du "Franc-Parleur 》.

"L'Evénement " et là question Lépine - Luigi. 27 nov. 1874.

Un discours au Parlement local-Luigi. 15 déc. 1874.

Quels sont les ennemis des catholiques - Luigi. 31 déc. 1874.

Ma marotte et leurs marottes - Luigi. 15 janv. 1875.

M. Fabre sénateur. Les travaux de nos ministres - Luigi. 10 fév. 1875.

L'Amnistie n'est pas l'amnistie. Luigi. 17 fév. 1875.

Le Vote sur la motion McKenzie - Luigi. 20 fév. 1875.

Nos deputés canadiens-français - Luigi. 10 mars 1875.

La Session - Luigi. 15 avril 1875.

Le Liberalisme politique - Luigi. 20 avril 1875.

La Religion des Libéraux et des Rouges - Luigi. 23 avril 1875.

Le Prêtre et la politique - Luigi. 28 avril 1875.

Quelques fredaines de "l'Evénement 》 - Luigi. 30 avril 1875.

[Article sans titre] - Luigi. 4 mai 1875.

Ne sachant que répondre, ils se fâchent et mentent - Luigi. 4 mai 1875.

Le Parti de la réforme - Luigi. 21 mai 1875.

Le "Journal de Québec " et "l'Evénement " contre le mandement de Mgr de Montréal Luigi. 4 juin 1875.

Fourberie des liberaux - Luigi. 9 juin 1875.

L'Eglise libre dans un Etat libre - Ste. Foi. 25 juin 1875.

Le Duo Fabre-Cauchon - Luigi. 25 juin 1875.

Les Juifs - Ste-Foi. 1er juillet 1875.

Une prouesse de l'Evénement - Luigi. 1er juillet 1875.

M. l'Abbé Sax et ses souffleurs - 31 aoat, 3, 8, 10 et 14 sept. 1875.

N.B. - Mis en brochure.

Doutre et Guibord - Luigi. 10 sept. 1875.

Ce que valent quelques affirmations et citations de "l'Evenement - Luigi. 1er oct. 1875.

Le “ Courrier du Canada " et "l'Evénement "-Luigi. 8 oct. 1875.

[ Article sans titre ] - Luigi. 12 oct. 1875.-

Un mot au "Courrier du Canada " - Luigi. 19 oct. 1875.

Ce que nous répond le * Courrier du Canada \- Luigi. 27 oct. 1875.

A propos de la dïme - Luigi. 19 nov. 1875.

Immunités ecclésiastiques - Luigi. 30 nov. 1875.

Libéralisme et franc-maçonnerie - Luigi. 11 déc. 1875.

Au "Bien Public " - Luigi. 17 déc. 1875.

La Franc-Maşonnerie - Luigi. 24 déc. 1875.

Le "Bien Public " et les immunites - Luigi. 7 janv. 1876.

Un mot de l'influence indue - Luigi. 5 fév. 1876.

La Lettre de Mgr Lynch à M. McKenzie - Luigi. 9 fév. 1876.

Mgr Gaume - Luigi. 19 fév. 1876.

Liberaux-Protestants - Luigi. 25 fév. 1876. 
La Dernière lettre pastorale de Mgr de Montréal attaquée par M. Galt - Luigi. 7 mars 1876.

Ce qui excuse le "Bien Public ", "l'Evenement " et beaucoup d'autres - Luigi. 14 mars 1876.

Les Seigneurs de l'épée et les Seigneurs de la plume - Luigi. 28 mars 1876.

$M$. Langelier et M. Gambetta - Luigi. 31 mars 1876.

Durera-t-il longtemps? - Luigi. 4 avril 1876.

Libéralisme - Luigi. 18 avril 1876.

N.B. - Cet article et ceux qui suivirent 21, 25, 29 avril; 2, 5, 9, 12, 16, 24, 30 mai; 9,14 , et 21 juin, furent mis en brochure sous le titre: Coup d'œil sur le libéralisme europeen et sur le libéralisme canadien.

La Lettre de Mgr Lynch à Sir Galt - Luigi. 25 avril 1876.

Un des plus dévots du parti liberal - Luigi. 9 mai 1876.

A propos de l'opinion du Dr de Angelis - Luigi. 19 mai 1876.

Le Sermon prêché à la Malbaie par le Rév. A. Pelletier - Luigi. 15, 18 et 22 aoùt 1876.

"L'Evénement " du 26 juillet contre Luigi - Luigi. 25, 29 aout et 1er sept. 1876.

L'Université Laval - Luigi. 8 sept. 1876.

La Circulaire de feu Mgr Baillargeon en date du 12 août 1868 a-t-elle provoqué un "monitum" du Saint-Office? - Luigi. 19 sept. 1876.

Le Triomphe de "L'Evénement" à propos de l'érection canonique de l'Université Laval - Luigi. 26, 29 sept., et 3 oct. 1876.

$A$ propos du " monitum " - Luigi. 26 sept. 1876.

Un nouveau bref de Pie IX condamnant les Catholiques libéraux - Luigi. 13 oct. 1876.

Quelque chose d'amusant - Luigi. 17 oct. 1876.

Quelques reflexions sur le dernier Bref de Pie IX - Luigi. 24 oct. 1876.

Immunites personnelles de $M$. le professeur Langelier et de ses amis - Luigi. 3 nov. 1876.

Le Liberalisme canadien condamné par Pie IX - Luigi. 3 nov. 1876.

L'Événement - Luigi. 7 nov. 1876.

La Plaidoirie de Charlevoix - Luigi. 10, 21, 24 nov.; 1er et 7 déc. 1876.

Le Suprême effort des libéraux - Luigi. 14 et 17 nov. 1876.

Le Jugement de Son Honneur le Juge Routhier dans la cause de Charlevoix - Luigi. 12 nov. déc. 1876.

Reponse à "Rétribution" - Luigi. 15, 19, 22 et 28 déc. 1876.

Un avertissement du St. Office à George St. Aimé - Luigi alias Alexis Pelletier, Ptre. 19 janv. 1877.

\section{La Minerve (Montreal)}

Correspondance - Alexis Pelletier, Ptre. 12 juillet 1881.

Correspondance - Alexis Pelletier, Ptre. 18 juillet 1881.

V L'Étendard (Montréal)

Correspondance - Alexis Pelletier, Ptre. 9 mai 1887. 